\title{
ONDE ESTÁA ENFERMAGEM? A (IN)VISIBILIDADE DESTA CATEGORIA PROFISSIONAL NOS MEIOS DE COMUNICAÇÃO
}

João Caio Silva Castro Ferreira ${ }^{1}$ Hylda Mara Cruz de Moraes ${ }^{1}$ Francisco José de Araújo Filho ${ }^{1}$ Maria Karolayne de Araujo Pereira ${ }^{1}$ Marcos Renato de Oliveira ${ }^{22}$

\author{
https://orcid.org/0000-0003-3497-5896 \\ https://orcid.org/0000-0003-4703-7081 \\ https://orcid.org/0000-0002-4203-7720 \\ https://orcid.org/0000-0002-9915-6843 \\ https://orcid.org/0000-0003-1803-9870
}

Objetivo: analisar a visibilidade da Enfermagem nos meios de comunicação segundo a percepção de acadêmicos de Enfermagem. Método: trata-se de uma pesquisa descritiva e exploratória, com abordagem qualitativa, com a participação de acadêmicos de Enfermagem do primeiro e último semestres. Concebeu-se a coleta de dados por meio de roteiro semiestruturado. Analisaram-se os dados sob os preceitos da Análise de Conteúdo associada com o software Interface de R pour lês Analyses Multidimensionalles de Textes et de Questionnaires (IRAMUTEO)®. Resultados: destacou-se, pelos entrevistados, que a Enfermagem é desvalorizada nos meios de comunicação, salientando, também, a recorrente valorização dos profissionais da Medicina nessas mídias em detrimento da Enfermagem e demonstrando a necessidade de reivindicar esta realidade. Conclusão: cerca-se a Enfermagem de estereótipos em sua representação nas mídias e isso implica a sua desvalorização social e visibilidade profissional.

Descritores: Midia Audiovisual; Papel do Profissional de Enfermagem; Autoimagem; Valorização Social.

\section{WHERE IS NURSING? THE (IN)VISIBILITY OF THIS PROFESSIONAL CATEGORY IN THE MEDIA}

Objective: to analyze the visibility of Nursing in the media according to the perception of Nursing students. Method: it is a descriptive and exploratory research, with a qualitative approach, with the participation of nursing students from the first and last semesters. Data collection was conceived using a semi-structured script. Data was analyzed under the precepts of Content Analysis associated with the software Interface de $R$ pour lês Analyses Multidimensionalles de Textes et de Questionnaires (IRAMUTEQ)®. Results: it was highlighted, by the interviewees, that Nursing is devalued in the media, also emphasizing the recurrent valorization of Medicine professionals in these media to the detriment of Nursing and demonstrating the need to claim this reality. Conclusion: stereotypes Nursing is surrounded in its representation in the media and this implies its social devaluation and professional visibility.

Descriptors: Audiovisual Media; Role of the Nursing Professional; Self-image; Social Appreciation.

\section{¿DÓNDE ESTÁ ENFERMERÍA? LA (IN)VISIBILIDAD DE ESTA CATEGORÍA PROFESIONAL EN LOS MEDIOS}

Objetivo: analizar la visibilidad de Enfermería en los medios de comunicación según la percepción de los estudiantes de Enfermería. Metodo: se trata de una investigación descriptiva y exploratoria, con enfoque cualitativo, con la participación de estudiantes de Enfermería del primer y último semestre. La recolección de datos se concibió utilizando un guión semiestructurado. Los datos se analizaron bajo los preceptos del Análisis de Contenido asociado con el software Interface de $R$ pour lês Analyses Multidimensionalles de Textes et de Questionnaires (IRAMUTEQ) ®. Resultados: los entrevistados destacaron que la Enfermería se devalúa en los medios de comunicación, destacando también la valoración recurrente de los profesionales de la Medicina en estos medios en detrimento de la Enfermería y demostrando la necesidad de reclamar esta realidad. Conclusión: la Enfermería está rodeada de estereotipos en su representación en los medios y esto implica su devaluación social y visibilidad profesional. Descriptores: Medios Audiovisuales; Rol del Profesional de Enfermería; Autoimagen; Apreciación Social.

'Universidade Federal do Piaui (UFPI)- Campus Senador Helvidio Nunes de Barros.

${ }^{2}$ Universidade Estadual do Ceará-UECE.

Autor Correspondente: João Caio Silva Castro Ferreira Castro Silva - Email: joaovscaiovscastro@outlook.com

Recebido 19/12/2019 - Aceito 31/03/2020 


\section{INTRODUÇÃO}

No século XXI, foram presenciadas diversas mudanças socioeconômicas que estimularam a produção de bens de consumo e serviços para a população. Em associação a tal fato, a popularização das Tecnologias Digitais de Informação e Comunicação (TDIC) permitiu o aumento da troca de informações e de acesso às mesmas ${ }^{(1)}$, influenciando, inclusive, as relações no setor de saúde humana e os profissionais envolvidos no cuidar.

A interatividade deste cenário tecnológico com a disseminação da imagem de alguns profissionais, como os enfermeiros (as), trouxe à tona o fato de que a Enfermagem pouco tem aproveitado seu espaço na mídia para repercutir sua verdadeira importância, submetendo-se ao modelo biologicista, hospitalocêntrico e submisso à Medicina(2).

É pertinente ressaltar que a Enfermagem, como ciência do cuidado, exerce ações de promoção, prevenção e reabilitação à saúde. A graduação em Enfermagem capacita os alunos a se tornarem futuros profissionais proativos e capazes de administrar seus serviços e promover a assistência apropriada à sua demanda populacional de acordo com suas peculiaridades e necessidades ${ }^{(3)}$.

O que se percebe é que a Enfermagem é uma profissão cada vez mais diversa no seu campo de atuação, todavia, os rótulos históricos ainda estigmatizam a profissão e limitam a disseminação do seu potencial (4), contribuindo, assim, para a disseminação de estereótipos e opiniões negativas nas mídias ${ }^{(5)}$

Um dos principais rótulos agregados à Enfermagem nas mídias de cinema e televisão é a imagem de profissionais do sexo feminino usando trajes brancos e obedecendo a ordens da equipe médica. Dessa maneira, as mídias insistem em associar a Enfermagem a rótulos sexistas e hospitalocêntricos que, em sua totalidade, não representam a realidade da Enfermagem e muito menos estão adequados ao retrato social destes profissionais ${ }^{(6)}$

Além dos pontos destacados anteriormente como justificativa para este estudo, a Enfermagem possui um grande quantitativo de profissionais no Brasil. Existem atualmente 2.223.194 profissionais de Enfermagem, contando-se entre auxiliares de Enfermagem, técnicos, enfermeiros (as) e obstetrizes $^{(7)}$. Além disso, o censo recente aponta que existiam 119.753 vagas em instituições públicas e privadas para o curso de Enfermagem ${ }^{(8)}$. Porém, o número de estudos sobre a representação social da Enfermagem, principalmente nas mídias televisivas, ainda é escasso.

Assim, este estudo torna-se relevante por apresentar a representação da Enfermagem, nos meios de comunicação, uma vez que os ideais difundidos podem influenciar toda a categoria. Justifica-se ainda a realização do mesmo para a obtenção de maior visibilidade científica e como forma de des- mitificar a imagem profissional destes indivíduos.

Dessa forma, esta pesquisa buscou analisar a visibilidade da Enfermagem nos meios de comunicação segundo a percepção de acadêmicos de Enfermagem.

\section{MÉTODO}

\section{Tipo de estudo}

Trata-se de uma pesquisa descritiva e exploratória, com abordagem qualitativa. Com este modelo de pesquisa, buscaram-se descrever populações distintas ou algumas situações ocorridas, buscando reunir ideias sobre acontecimentos notificados. Além disso, também objetivou-se ressaltar e mudar conceitos pré-estabelecidas, pretendendo-se erguer outras indagações para novas pesquisas ${ }^{(9)}$.

\section{Participantes da pesquisa}

Para participar deste estudo, foram escolhidos 22 estudantes de Enfermagem cursando o primeiro ou o último semestre da graduação. A amostra é composta por 15 acadêmicos (as) de Enfermagem no último semestre de graduação e 18 pessoas eram do sexo feminino. Os critérios de inclusão da pesquisa foram: graduandos do curso de Enfermagem cursando o primeiro ou o último semestre da graduação, com idade superior a 18 anos, que concordassem em ser entrevistados e que estivessem regularmente matriculados na Instituição de Ensino Superior (IES) em que foi realizada a pesquisa. Já os critérios de exclusão foram: alunos que não se encaixavam no perfil mencionado anteriormente e que apresentassem alguma condição física que não permitisse a comunicação.

\section{Local do estudo}

O respectivo estudo foi realizado no período de setembro a novembro de 2017 em uma IES pública situada em Picos, interior do Piauí. A seleção da instituição foi intencional pelo fácil acesso à população amostral, além de ser o estabelecimento de ensino ao qual o pesquisador estudava, favorecendo a etapa de coleta dos dados.

\section{Coleta de dados}

Como ferramenta para a coleta de dados, foi utilizada a entrevista semiestruturada previamente marcada com os entrevistados e realizada em um local que assegurasse a privacidade dos mesmos. Ela foi conduzida por um roteiro construído pelos autores. Este instrumento possui uma pergunta discursiva e três objetivas no qual se buscou conseguir um detalhamento do perfil dos estudantes que adentraram no estudo. Para a gravação da entrevista, foi usado um software android 4.1.2 Jelly Bean. O questionamento central do roteiro foi: "O que você acha da forma que a Enfermagem é representada nos meios de comunicação?". 


\section{Procedimentos de análise dos dados}

A análise dos dados foi concebida pela aplicação da Análise de Conteúdo associada ao software IRAMUTEQ®. versão 0.7 alpha 2. O procedimento de análise seguiu as respectivas etapas: pré-análise; investigação do material e tratamento dos resultados; inferência e interpretação(10).

A Análise de Conteúdo foi empregada com o objetivo de designar afinidades entre os arranjos semânticos ou linguísticos, assim como entre as estruturas de cunho psicossociais. Este segmento analítico baseia-se na desintegração da unidade textual, buscando-se localizar os núcleos secundários incorporados na comunicação dos ouvintes e, subsequentemente, realizar a reunião em classes ou categorias das ideias que expressam mensagens similares ${ }^{(10)}$.

Como suporte na análise de dados, o software IRAMUTEQ ${ }^{\circledR}$ possibilitou diferentes análises estatísticas de textos $^{(11)}$. É válido salientar que esse software pode realizar diversos tipos de análises textuais dos quais, para este estudo, foi utilizado somente a técnica de nuvem de palavras. A nuvem agregou as palavras dos materiais analisados e as dispõe graficamente em função da sua frequência(12).

\section{Procedimentos éticos}

Este estudo faz parte de uma pesquisa maior: Influência das séries de televisão na representação do papel da enfermagem. A mesma foi aprovada por um Comitê de Ética em Pesquisa (CAAE: 70797517.4.0000.8057), em conformidade com as normas impostas na resolução $n^{\circ} 466$, de 12 de dezembro de 2012, do Conselho Nacional de Saúde, e obteve o parecer de aprovação com o número 2.269.337. Além disso, antes da coleta de dados, foi feita a leitura do Termo de Consentimento Livre e Esclarecido (TCLE) onde se apresentaram detalhes sobre a pesquisa e os direitos que foram contemplados para os seus sujeitos que aceitaram ser entrevistados. Para garantir o sigilo das entrevistas transcritas no estudo, elas foram codificadas com a letra $E$ seguida de seu número de ordem.

\section{RESULTADOS}

A análise dos resultados gerou três categorias: Os cenários de desvalorização da Enfermagem; Reivindicação pela valorização da Enfermagem nos meios de comunicação e Visão biomédica nas mídias.

\section{Os cenários de desvalorização da Enfermagem}

Para compreender a motivação deste processo de descaso com a Enfermagem, foram listadas, pelos entrevistados, três subcategorias que se correlacionam com este ideal: as desvalorizações midiática, política e financeira, como apresentadas a seguir.

\section{Subcategoria 1: Desvalorização midiática}

Péssima, uma classe que não tem destaque que realmente merecia ter. (EII)

Eu vejo a Enfermagem como uma profissão esquecida porque retrata apenas a questão do enfermeiro, como (...) auxiliar, os demais profissionais, sendo que a profissão da Enfermagem é uma pro-

fissão independente. (El4)

$E$ as pessoas acabam que quem assiste (televisão) acha que a gente não faz nada porque não aparece na televisão, então, se não aparece na televisão, não faz. (E2)

\section{Subcategoria 2: Desvalorização política}

Precisa de uma visibilidade maior tanto dos políticos. (E8)

[...] sendo que o verdadeiro culpado nem somos nós, na verdade, é o Estado. (E7)

\section{Subcategoria 3: Desvalorização financeira}

Porque o salário de Enfermagem não dá dinheiro. (E10) Também até questão do salário, que é diferente do médico, pouco valorizada neste quesito. (E5)

Subentende-se que os diferentes parâmetros de desvalorização apresentados motivam a invisibilidade da Enfermagem, fragilizando a sua repercussão social. Todavia, esta fragilidade é utilizada como viés para explorar negativamente a imagem social da Enfermagem, criando-se um distanciamento entre o reconhecimento da Enfermagem e a alta repercussão de preconceitos e estereótipos que não condizem com a realidade profissional.

Somente uma pessoa, que integrava o último semestre da graduação, divergindo da maioria representativa que alegou a invisibilidade da Enfermagem, mencionou a valorização da Enfermagem nos meios de comunicação, como pode ser notado a seguir.

[...] eu acho que hoje as pessoas têm uma visão mais aberta e que elas têm uma noção maior da importância da Enfermagem, pelo menos, nos meios de comunicação, tipo Facebook, Instagram que eu sigo, eu vejo a Enfermagem de uma forma positiva e de uma maneira indispensável no cuidado, eu acho

que eles são bem representados, apesar de que, claro, tem as exceções, mas, no geral, eu acho que a Enfermagem e bem representada. (Ell) 
Apesar da grande invisibilidade ainda existente na profissão de Enfermagem, pode-se verificar que, aos poucos, os profissionais e acadêmicos começam a ocupar espaço positivo na mídia, espaço este que vem sendo utilizado para disseminar os campos práticos, as competências e os conhecimentos científicos em que se baseia a atuação da Enfermagem.

Reivindicação pela valorização da Enfermagem nos meios de comunicação

Tal categoria mostra que, mesmo neste contexto de invisibilidade e desvalorização, existe a intenção dos acadêmicos em contestar essa situação e romper com esse quadro de preconceitos históricos em torno da Enfermagem, como se pode perceber por meio dos recortes de discurso apresentados a seguir.

E eu acho que seria de suma importância que a Enfermagem fosse mais representada... (E1O)

O enfermeiro que tá ali sempre presente, [enf.] tem o primeiro contato com o paciente, ele que vai elaborar todos os tipos de cuidados e vai observar, então, o enfermeiro tem que ganhar sim sua importância. (El3)

O enfermeiro tem uma importância muito grande dentro de todos os procedimentos, praticamente o enfermeiro está dentro de todos os procedimentos, então, eu acho que a gente deveria ser mais representados nos meios de comunicação. (EOI)

É neste cenário que se percebe a insatisfação dos acadêmicos perante a invisibilidade da Enfermagem nos meios de comunicação, afinal, segundo seus conhecimentos, sabe-se que a assistência de Enfermagem possui grande impacto no sistema de saúde do Brasil. Dessa forma, a Enfermagem merece mais destaque, porém, tal realidade ainda não é devidamente reconhecida e popularizada pelas midias.

Não obstante, por meio da nuvem de palavras gerada (Figura 1), foi possível ilustrar como é a representação da Enfermagem nos meios de comunicação segundo os acadêmicos entrevistados. O corpus textual analisado reuniu 308 palavras. Destas, pode-se perceber, por meio da nuvem, quais palavras foram mais destacadas nos discursos dos sujeitos.
Figura 1- Nuvem de palavras sobre a representação da Enfermagem nos meios de comunicação segundo a opinião dos acadêmicos de Enfermagem. Picos, Piauí, Brasil, 2017.

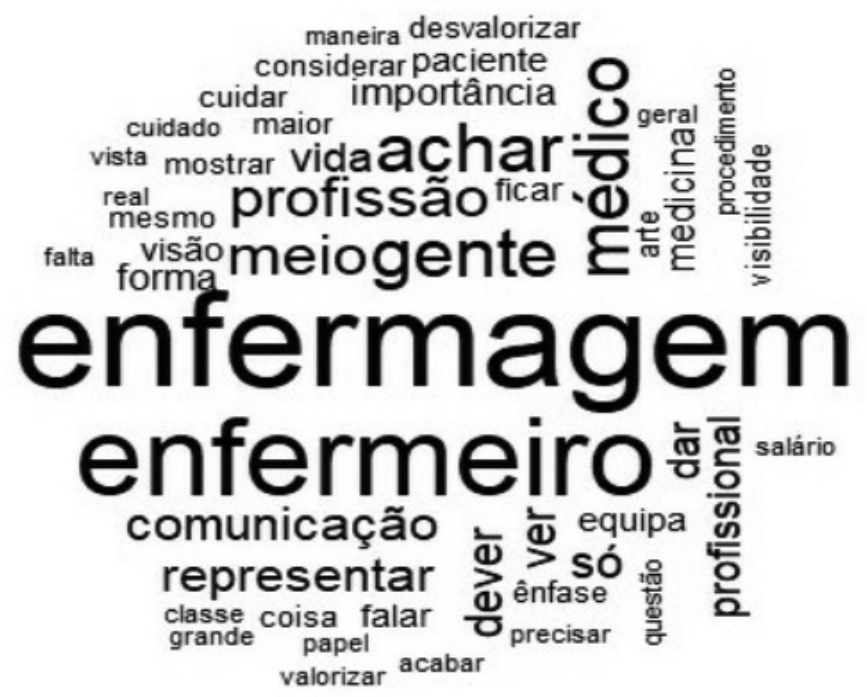

A nuvem de palavras demonstra que a palavra Enfermagem se encontra em evidência por ser a palavra mais evocada (32 vezes), mas se pode notar que outras palavras também tiveram ênfase e, dentre elas, há: Comunicação; Gente; Médico; Meio e Enfermeiro. Perante estes destaques, pode-se perceber o quanto o processo de Enfermagem é um processo grandioso, que requer comunicação minuciosa com os demais profissionais, em especial, os profissionais médicos e os pacientes.

Visão biomédica nas mídias

A Enfermagem é bastante desvalorizada nos meios de comunicações e comparada à classe médica ao ponto que, segundo os entrevistados, estes meios somente representam o setor saúde com foco nos profissionais da Medicina, como pode ser visto a seguir.

Acho que a ênfase maior ela tá sendo na Medicina do que na Enfermagem. (E2I)

Eles sempre dão ênfase ao trabalho realizado pelos médicos, subjugando a Enfermagem como menos importante. (E15)

Foi identificado, nas falas dos entrevistados, o quanto a Enfermagem é cercada de estereótipos e vista de forma distorcida pelos meios de comunicação. A invisibilidade histórica da Enfermagem ainda é refletida no cotidiano, todavia, a profissão ainda não alcançou o seu patamar de protagonismo como ciência do cuidado, o que requer mais 
empenho desta categoria profissional para atingir seu devido reconhecimento.

\section{DISCUSSÃO}

Esse estudo revelou o quanto a Enfermagem é reconhecida por seus estereótipos históricos, entre eles, a inferiorizarão da Enfermagem perante a Medicina. Além disso, pode-se perceber o quanto a profissão possui pouca visibilidade nos meios de comunicação. Sabe-se que a Enfermagem evoluiu perante a importância da sua assistência, mas a mídia ainda não conseguiu acompanhar essa realidade ${ }^{(13)}$ e, para entender essa insistente representação estereotipada da Enfermagem, precisam-se compreender o cotidiano da profissão e quais as dificuldades que influem na persistência destes rótulos.

Esse cotidiano profissional é marcado por condições de trabalho insuficientes, estresse, falta de competência na equipe e excesso de trabalho. E tal realidade sobrecarrega o trabalho do (a) enfermeiro (a), além de precarizar o trabalho da Enfermagem, provocando o sofrimento moral dos seus profissionais. Portanto, as consequências dessa precarização interferem diretamente na perda de identidade e na satisfação em executar suas responsabilidades, o que implica o posicionamento profissional da Enfermagem perante a visibilidade da profissão ${ }^{(14-16)}$.

Quando os profissionais de Enfermagem se sobrecarregam de responsabilidades, na tentativa de buscar mais credibilidade para seu trabalho, estes indivíduos descentralizam suas ações com base na sua prática profissional, agregando, ao seu trabalho, até mesmo funções que não são de sua responsabilidade, e é neste afastamento dos princípios da Enfermagem que emergem os sentimentos de descontentamento, frustrações e silenciamento perante a importância científica da Enfermagem como ciência do cuidado(17).

Ao que se refere às representações sociais categorizadas neste estudo, podem-se perceber os cenários de desvalorização da Enfermagem e a prevalência do modelo biomédico nos meios de comunicação, assim como tornou-se evidente a intenção dos acadêmicos de Enfermagem em romper com esta realidade e elevar a Enfermagem para um patamar em que seja reconhecida socialmente a importância da profissão. Além disso, vale destacar que a opinião sobre a desvalorização da Enfermagem foi dominante tanto para alunos do primeiro semestre quanto para alunos do último semestre de graduação.

Conquanto este cenário de desvalorização pode ser percebido em vários aspectos sociais, nos meios de comunicação da área de saúde ${ }^{(18)}$ e também nas demais mídias, em uma pesquisa para investigar o nível de atenção aos profissionais da Enfermagem nas mídias portuguesas, foram recolhidas 1.271 notícias específicas da área da saúde. Contudo, entre os profissionais que foram fontes de informação para a construção de alguma matéria sobre saúde, somente $6,6 \%$ da amostra foi constituída por profissionais da Enfermagem, enquanto o profissional médico teve o índice de $22,5 \%{ }^{(19)}$.

Tal fato destaca o respaldo social que a Medicina possui. Pode-se compreender que os profissionais médicos possuem maior credibilidade para se posicionar nos meios de comunicação, repercutindo informações que se concentram na visibilidade de somente um profissional e, consequentemente, os telespectadores passaram a dar credibilidade apenas às profissões que se pronunciam socialmente, enquanto as que se mantêm em silêncio não são validadas.

Ainda assim, é importante que a equipe de Enfermagem rompa com este silêncio perante suas práticas e fortaleça suas aptidões comunicacionais e organizacionais, visando à melhoria da sua contribuição no espaço público. Dessa maneira, a sociedade poderá identificar os progressos da profissão, a sua missão social e as suas colaborações para o sistema de saúde vigente, posteriormente, fortalecendo a importância da Enfermagem para o setor social e para os meios de comunicação(19).

Uma alternativa para aumentar a visibilidade da Enfermagem são as redes sociais, como o Facebook, Instagram e Twitter, pois, além de possuírem ferramentas para promover reflexões sobre a importância da Enfermagem, são recursos bastante explorados pelos discentes, docentes e os demais graduados em Enfermagem ${ }^{(20-21)}$. Dessa forma, os diálogos em torno da importância da Enfermagem são popularizados e, aos poucos, ganham notoriedade ${ }^{(22)}$.

Como iniciativa mobilizadora para fornecer mais visibilidade no campo social e nas políticas públicas para a Enfermagem, instâncias governamentais internacionais criaram a Campanha Nursing Now. A mesma possui cunho mundial e foi inaugurada em fevereiro de $2018^{(23)}$. Neste contexto, percebe-se que iniciativas fortes finalmente estão começando a surgir para ressalvar a importância da Enfermagem, porém, muito ainda deve ser feito para que a profissão tenha seu devido respaldo sociopolítico.

Diante do exposto, constatou-se que a Enfermagem, de forma gradativa, está se organizando para romper os estereótipos históricos que the cercam, contudo, ainda se tem um longo caminho para ser percorrido e a soma de esforços, associada à conscientização social, constitui os primeiros passos para ascender a Enfermagem a um estágio superior.

\section{Limitações do estudo}


Uma das principais limitações desta pesquisa foi a escassez de materiais científicos que agregassem conhecimentos sobre a temática debatida. Além disso, é pertinente relatar a dificuldade para acessar sujeitos de grupos distintos para uma mesma pesquisa, tornando-se cruciais o planejamento e o diálogo para marcar as entrevistas de acordo com a disponibilidade de cada entrevistado associada ainda à dificuldade de encontrar literatura para melhor comparar os achados deste estudo.

\section{Contribuição para a prática}

A contribuição deste estudo concentra-se na sua proposta em alertar os profissionais da Enfermagem sobre como a importância profissional de sua categoria está sendo despercebida pelos meios de comunicação, fato que deve ser reivindicado para que a Enfermagem conquiste seu devido reconhecimento nos âmbitos socioeconômico e político.

\section{CONCLUSÃO}

Diante dos resultados encontrados neste estudo, pode-se concluir que a invisibilidade da Enfermagem se dá pela restrita quantidade de profissionais que utilizam as mídias sociais para a divulgação do trabalho desenvolvido, bem como a imagem errônea repassada pelos meios de comunicação

No que se refere à valorização da Enfermagem, verificou-se que, apesar da evolução conquistada, esta categoria ainda é vista como submissa ao ato médico, além da discrepante diferença salarial equiparada à carga do trabatho desenvolvido, o que contribui significativamente para a desvalorização da profissão.

Um meio disponível para facilitar a visibilidade e promover a valorização são as redes sociais, ferramentas cruciais para a repercussão de novas informações, entretanto, os profissionais e acadêmicos de Enfermagem devem empoderar-se sobre como usar estas tecnologias a favor do reconhecimento da Enfermagem.

De tal forma, convocam-se pesquisadores, sociedade civil e entidades de classe, bem como enfermeiros e enfermeiras assistenciais, a estarem mais atentos às representações midiáticas da classe de Enfermagem e assim cobrar, dos meios de comunicação, que essa visibilidade seja justa às ações desenvolvidas pela Enfermagem.

\section{Contribuição dos autores}

Autores 1 e 5: concepção e/ou desenho; análise; interpretação dos dados; redação do artigo; revisão crítica e a revisão final. Autores 2, 3 e 4: revisão crítica e revisão final.

\section{REFERÊNCIAS}

1. Pereira CA. A midia na Ciência da Informação. TransInformação. [Internet]. 2018 [Cited 2019 Jul. 04];30(2):141-152. Available from: http://dx.doi.org/10.1590/2318-08892018000200001

2. Fonseca LF, da Silva MJP. Desafiando a imagem milenar da enfermagem perante adolescentes pela internet: impacto sobre suas representações sociais. Cienc Cuid Saúde. [Internet]. 2012[Cited 2019 Jul. 04]; 11(suplem.):054-062. Available from: http://dx.doi.org/10.4025/cienccuidsaude.v1li5.17052

3. Tanaka EZ, Sartori DVB, Ferreira LR, Bermejo LJ. A educação a distância nos cursos de graduação em enfermagem: aplicação e efetividade. Revista online de Política e Gestão Educacional. [Internet].2017 [Cited 2019 Jul. 04 ]; 21 (esp. 1): 831-841.Available from: https://periodicos.fclar.unesp.br/rpge/article/view/10455

4. Silva RG, Sant'Anna AS. Trajetórias de carreira na enfermagem: uma análise com profissionais em distintos grupamentos etário-geracionais. Rev gest sist Saúde.[Internet]. 2017 [Cited 2019 Jul. 04];6(2): 100-113.Available from: http://www.revistargss.org.br/ojs/index.php/rgss/article/view/319

5. Calvo-Calvo MA. Sexist stereotypes and biasesassociated with the female nursing model in advertising communication. Texto \& contexto Enferm. [Internet]. 2014 [Cited 2019 Jul. 04]; 23(3): 530-537. Available from: http:// dx.doi.org/10.1590/0104-07072014004120012

6. Calvo-Calvo MA. Imagen social de las enfermeras y estrategias de comunicación pública para conseguir una imagen positiva. Index Enfer.[Internet]. 2011 [Cited 2019 Jul. 04]; 20(3): 184-188. Available from: http://dx.doi.org/10.4321/ S1132-12962011000200010

7. Conselho Federal de Enfermagem. Enfermagem em Números. Disponível em: http://www.cofen.gov.br/enfermagemem-numeros . Acesso em: 25 de março de 2019 
8. Teixeira E, Fernandes JD,Andrade AC, Silva KL, Rocha MEMO, Lima RJO. Panorama dos cursos de Graduação em Enfermagem no Brasil na década das Diretrizes Curriculares Nacionais. Rev Bras Enferm. [Internet]. 2013 [Cited 2019 Jul. 04];66(esp):102-110.Available from: http://dx.doi.org/10.1590/S0034-71672013000700014

9. Gil, AC. Métodos e técnicas de pesquisa social.6. ed.São Paulo: Atlas, 2010.

10. Bardin, L. Análise de Conteúdo. Lisboa: Edições 70, 2011. 229 p.

11. Moura LKB, Marcaccini AM, Matos FTC, Sousa AFL.; Nascimento GC, Moura MEB. Revisão Integrativa sobre o câncer bucal. J.res.: fundam. Care. [Internet]. 2014 [Cited 2019 Jul. 04]; 6(supl):164-175.Available from: https://www. redalyc.org/html/5057/505750772020/

12. Kami MTM, Larocca LM, Chaves MMN, Lowen IMV, Souza VMP, Goto DYN. Trabalho no consultório na rua: uso do software IRAMUTEQ no apoio à pesquisa qualitativa. Esc Anna Nery. [Internet]. 2016 [Cited 2019 Jul. 04];20(3): e20160069.Available from: http://dx.doi.org/10.5935/1414-8145.20160069.

13. Gill J, Baker C. The Power of Mass Media and Feminism in the Evolution of Nursing's Image: A Critical Review of the Literature and Implications for Nursing Practice. J Med Humanit.[Internet]. 2019 [Cited 2019 Oct 27]. Available from: https://www.ncbi.nlm.nih.gov/pubmed/31713004

14. Wachholz A, Dalmolin GL, Silva AM, Andolhe R, Barlem ELD, Cogo SB. Sofrimento moral e satisfação profissional: qual a sua relação no trabalho do enfermeiro?. Rev. esc. Enferm. USP. [Internet].2019 [Cited 2019 Oct 27];53: e3510. Available from: http://dx.doi.org/10.1590/s1980-220x2018024303510

15. Pérez Junior EF, David HMSL.Trabalho de enfermagem e precarização: uma revisão integrativa. Enferm Foco. [Internet]. 2018 [Cited 2019 Jul. 04]; 9 (4): 71-76.Available from: http://revista.cofen.gov.br/index.php/enfermagem/ article/view/1325

16. Araujo CAS. The nursing professional and creation of values in health [editorial]. Acta Paul Enferm. [Internet]. 2016 [cited 2019 Oct 27]; 29(4):3-4. Available from: http://dx.doi.org/10.1590/1982-0194201600050

17. Fernandes MC, Silva LMS, Silva MRF, Torres RAM, Dias MSA, Moreira TMM. Identidade do enfermeiro na atenção básica: percepção do "faz tudo". Rev. Bras. Enferm.[Internet]. 2018 [Cited 2019 Sep. 25]; 71(1): 154-159, Available from: http://dx.doi.org/10.1590/0034-7167-2016-0382.

18. Mason D, Nixon L, Glickstein B, Han S, Westphaln K, Carter L. (2018). The Woodhull Study Revisited: Nurses Representation in Health News Media 20 Years Later. Journal of Nursing Scholarship. [Internet]. 2018 [Cited 2019 Oct 27] 50(6). Available from: https://doi.org/10.1111/jnu.12429

19. Cardoso RJM, Graveto JMGN, Queiroz AMCA. Visibilidade da enfermagem nas midias impressas e online. Rev Latino-Am Enferm. [Internet]. 2014 [Cited 2019 Jul. 04]; 22(1):1-6. Available from: http://www.scielo.br/pdf/rlae/ v22nl/pt_0104-1169-rlae-22-01-00144.pdf

20. Tuckett A, Turner C. Do you use social media? A study into new nursing and midwifery graduates' uptake of social media. Int J Nurs Pract. [Internet]. 2016 [Cited 2019 Oct 27] 22(2). Available from:https://www.ncbi.nlm.nih.gov/ pubmed/26531296

21. Mersin S, İbrahimoğlu Ö. Saray Kııç H, Bayrak Kahraman B. Social media usage alexithymia in nursing student. Perspect Psychiatr Care.[Internet].2019[Cited 2019 Oct 27]. Available from: https://www.ncbi.nlm.nih.gov/ pubmed/31680272

22. Kakushi LE, Évora YDM. As redes sociais na educação em enfermagem: revisão integrativa da literatura. Rev Latino-Am Enfermagem.[Internet]. 2016 [Cited 2019 Jul. 04]; 24:e2709.Available from: http://www.scielo.br/pdf/ rlae/v24/pt_0104-1169-rlae-24-02709.pdf.

23. Cassiani SHB, Lira Neto JCG. Perspectivas da Enfermagem e a Campanha Nursing Now. Rev. Bras. Enferm. [Internet]. 2018 [Cited 2019Jul. 04]; 71(5):2351-2352. Available from: http://dx.doi.org/10.1590/0034-7167.2018710501 